\title{
Deep Learning Technology: A Vital Tool for National Development
}

\author{
Agbaraji E.C \\ Computer Engineering Department, \\ Federal Polytechnic Nekede, \\ Owerri, Nigeria
}

\author{
Ihemereze C.N \\ Computer Engineering Department, \\ Federal Polytechnic Nekede, \\ Owerri, Nigeria
}

\begin{abstract}
This paper focuses on the study of deep learning technology as a vital tool for national development. There are many problems hindering the growth of most developing nations. Some of these problems have been related to the level of technological solutions available in such countries. Most economic, health and general social problems are now being tackled and solved using very high intelligent systems which can autonomously monitor and study a particular situation and provide the best decision that provides permanent solution to such problems. AI provides the basic intelligence of most systems and with deep learning which is a recent development in AI, a system can become very intelligent. From the review, it was concluded that deep learning can solve most of the challenges facing the developing nations.
\end{abstract}

Keywords-Deep learning; AI; machine learning; national development

\section{INTRODUCTION}

The technological world is very dynamic and actually evolving every day. The need for a continuous technological advancements to meet up with the customers desires and to solve some complex problems existing in the society which have not been addressed with conventional technology, has brought about the study of the deep learning technology which is a new area in machine learning which is a branch under artificial intelligent. This means that with the deep learning technology, the autonomous systems are made more intelligent and can now make better judgments and decisions when fed with natural data. Therefore, this will result to improvement in quality and quantity of most industrial products. It can also be applied in solving administrative and managerial problems especially when such problems are captured in data forms and the algorithm can be developed.

Deep Learning is a new area of Machine Learning research, which has been introduced with the objective of moving Machine Learning closer to one of its original goals: Artificial Intelligence [1]. It allows computational models that are composed of multiple processing layers to learn representations of data with multiple levels of abstraction. These techniques have dramatically improved the state-ofthe-art in speech recognition, visual object recognition, object detection and many other domains such as drug discovery and genomics. Even as machines known as "deep neural networks" have learned to converse, drive cars, beat video games and Go champions, dream, paint pictures and help make scientific discoveries, they have also confounded their human creators, who never expected so-called "deeplearning" algorithms to work so well. No underlying principle has guided the design of these learning systems, other than vague inspiration drawn from the architecture of the brain (and no one really understands how that operates either). Machine Learning is a subset of artificial intelligence. It allows the machines to learn and make predictions based on its experience (data) [2].

Artificial Intelligence (AI) is a technique which allows the machines to act like humans by replicating their behavior and nature [2]. It has provided tremendous solutions to support mankind in its activities because it provides the intelligence of autonomous machines and the improvement of cell phones and the internet. According to Meltzer [3], artificial intelligence stands to have transformative impact on international trade. Already, specific applications in areas such as data analytics and translation services are reducing barriers to trade. However, there are challenges in the development of AI that international trade rules could address, such as improving global access to data to train AI systems. The following provides an overview of some of the key AI opportunities for trade as well as those areas where trade rules can help support AI development.

National development cannot be achieved without the success of technological growth and its integration in the society. The economy of most developed nations is basically technologically driven and in order to maintain the growth of such economy, they continue to work towards using the most recent development. However, most developing countries continue to lag behind in economy and general development due to failure to keep up with the trend of technological development in the world. In most developing nations there exist a lot of problems which are hindering national growth such as fraud in banking system, lack of adequate security systems such as the cameras with high image processing and identification systems, lack of adequate industrial tools etc. The local innovations need to be improved on with the deep learning technology. These problems can be addressed by studying and implementing the deep learning technology.

The development challenges facing most developing nations are centered on management and administration. Most of the challenges and economic problems hampering the development of most developing countries can be solved through AI (deep learning) technology and the democratization of data which will lead to more transparent governance. This will empower the people to be active and understand what is going behind under the curtain. Furthermore, AI can improve various sectors and provide the edge that is needed, increasing efficient agricultural output discovering new niches and finally make the nation a diverse and competitive economy like developed nations [4]. 


\section{LITERATURE REVIEW}

Many research works have been carried out in the area of artificial intelligence, machine learning and deep learning. Table 1 shows the major AI domain. Schmidhuber [5] worked on Deep Learning in Neural Networks: An Overview. $\mathrm{He}$ stated that deep artificial neural networks (including recurrent ones) have won numerous contests in pattern recognition and machine learning in recent years. According to him, Shallow and deep learners are distinguished by the depth of their credit assignment paths, which are chains of possibly learnable, causal links between actions and effects. He reviewed deep supervised learning (also recapitulating the history of backpropagation), unsupervised learning, reinforcement learning \& evolutionary computation, and indirect search for short programs encoding deep and large networks. Wolchover [6] described it as a brain, a deep neural network has layers of neurons - artificial ones that are figments of computer memory. When a neuron fires it sends signals to connected neurons in the layer above. During deep learning, connections in the network are strengthened or weakened as needed to make the system better at sending signals from input data - the pixels of a photo of a dog, for instance - up through the layers to neurons associated with the right high-level concepts, such as "dog." After a deep neural network has "learned" from thousands of sample dog photos, it can identify dogs in new photos as accurately as people can. The magic leap from special cases to general concepts during learning gives deep neural networks their power, just as it underlies human reasoning, creativity and the other faculties collectively termed "intelligence." Experts wonder what it is about deep learning that enables generalization - and to what extent brains apprehend reality in the same way.

The concept of AI (deep learning) technology has many promises towards improving science and technology and also solves different social problems. Prince [4] argued that the impact of using this technology to develop humanitarian areas can have a multiplier effect in developing countries; some of these countries especially the developing nations possess abundant reserves of natural resources but lack the equipment and tools to explore them. By leveraging the power of $\mathrm{AI}$ and more importantly Deep Learning, businesses, nongovernmental organizations (NGOs) and governments can solve life-threatening, economical and technological problems to improve the wealth and livelihood of local communities in the developing world [4].

Copeland [7] stated that Artificial intelligence (AI) is the ability of a digital computer or computer-controlled robot to perform tasks commonly associated with intelligent beings. According to him the term is frequently applied to the project of developing systems endowed with the intellectual processes characteristic of humans, such as the ability to reason, discover meaning, generalize, or learn from past experience. Since the development of the digital computer in the 1940s, it has been demonstrated that computers can be programmed to carry out very complex tasks-as, for example, discovering proofs for mathematical theorems or playing chess - with great proficiency. Some programs have attained the performance levels of human experts and professionals in performing certain specific tasks, so that artificial intelligence in this limited sense is found in applications as diverse as medical diagnosis, computer search engines, and voice or handwriting recognition. Reycoso [8] in her work presented an extended description and various examples of present-day applications of AI as follows:

Artificial narrow intelligence (ANI): Artificial narrow intelligence, or also called weak $\mathbf{A} \mathbf{I}$, is a type of artificial intelligence that can only particularizes on one specific task or type of problem at a time. Narrow AI is programmed to complete a single task, such as forecasting the weather or playing a game. Narrow AI is neither self-aware nor sentient. Though it may seem highly capable, ANI is bound by strict programming for singular tasks. ANI is considered weak because it does not have the capacity to meet or exceed human intelligence or learn and adapt as other formats of AI can. Despite this, ANI machines may seem more knowledgeable and sophisticated by surpassing human knowledge or skill on the individual task for which it was programmed; however, these systems are operating as programmed, not because they are actively learning new information. An example of narrow AI is smartphone assistants like Bixby or Siri. Though they can "communicate" with human users, their responses are limited by a lack of understanding words and phrases beyond what they were programmed to interpret.

Artificial general intelligence (AGI): Artificial general intelligence (AGI), or strong $\mathbf{A I}$, is the opposite form of ANI. AGI refers to machines that can successfully perform human tasks. This type of intelligence is considered "humanlike," given that general AI can strategize, reason, learn, and communicate in a manner aligned with human functions and processes. In addition, some AGI machines are able to see (by means of computer vision) or manipulate objects. Currently, AGI is in preliminary stages with hypothetical real-life applications on the horizon in the foreseeable future.

Big data: Big data defines large amounts of structured and unstructured data. It is a field that analyzes and extracts information from mass amounts of information (data) that is too complex to be handled by standard data-processing software. An example of big data in product development is Netflix. Because Netflix's user base is at or beyond 100+ million people, they use big data to build predictive models to improve user experience. Whenever you get a recommendation about a show or movie that might interest you based on what you've watched before, Netflix is utilizing its mass amount of user data and preferences to curate a selection of likely matches for individual users. Netflix gathers big data in a multitude of ways by tracking how a user discovers a program or movie (search function, suggestion); star ratings; search queries; when or if users pause or stop watching a show/program; date(s) the content was watched; and more. They use this data to recommend new content to users and show a user "what's trending" (which may influence some users to watch to be in the know) with hot, new programs.

Computer vision: Computer vision is when a machine processes visual input from image files (JPEGs) or camera feeds. Not only can computer vision "see" the image(s), but it also understands and processes what it is seeing. If this were 
put in terms of human existence, computer vision is to brain comprehension as eyes are to seeing. Basically, whenever a machine processes raw visual input - such as a JPEG file or a camera feed - it's using computer vision to understand what it's seeing. It's easiest to think of computer vision as the part of the human brain that processes the information received by the eyes - not the eyes themselves. To simplify, utilizing computer vision means the user is inputting an image into the system, and what the user receives as an output can include quantitative and qualitative features of the image, including color, shape, size, and classification. An example of computer vision is the images that Tesla's self-driving cars see. The system has to not only recognize images by shape, type, and color, but also process these pieces of information extremely quickly given that it is performing an action in real-time.

Data mining: Data mining is the process of sorting through large sets of data in order to identify recurring patterns while establishing problem-solving relationships. Data mining is a blended subset of computer science and statistics whose sole purpose is to extract data using AI while turning it into useful information. Examples of data mining occur in e-commerce, with Amazon spearheading the data collection game. Amazon targets their customers and uses their data by showing buyers recommended products "others" have bought in relation to the consumer's intended purchase (i.e. if you're considering buying this, people usually also purchase that). Amazon uses customer data (what people purchased plus what people said about their purchases) to identify buying patterns and infer what customers may like based on other user data.

Machine learning: Machine learning focuses on developing programs that access and use data on their own, leading machines to learn for themselves and improve from learned experiences without explicitly being programmed. Many examples of machine learning in day-to-day life currently exist, including targeted advertisements on social media, virtual voice assistants on cell phones, facial recognition software on social media websites as demonstrated in figure 1 , and commuting predictions from apps like Google Maps or cellphone GPS data.

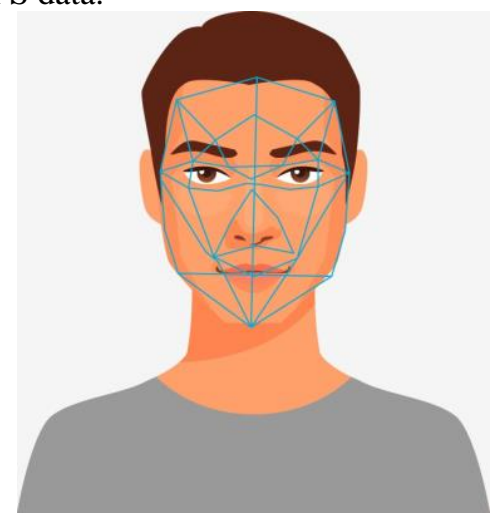

Figure 1: Image processing (Source: Image courtesy of vigilantsolutions.com)

Deep learning: Deep learning is a machine learning technique that teaches computers how to learn by rote. In other words, deep learning allows machines to gain the ability to mimic learning as a human mind would by classifying text, sound, and images into categories. Examples of deep learning are found in various existing technologies, such as driverless cars and voice assistants. These specific examples utilize deep learning techniques by learning from hundreds - if not thousands - of hours of video, images, and samples by which the technology self-teaches pattern recognition. For instance, driverless cars learn how to drive and navigate roads from studying road patterns and driving habits of existing human drivers and other vehicles. Similarly, voice assistants listen to endless hours of speech data from people with different voice types, languages, and speech patterns in order to learn how to replicate human speech.

Neural networks: A neural network models itself after the human brain by creating an artificial neural network via a pattern-recognizing algorithm. This algorithm allows a computer to learn from and interpret sensory data with the purpose of classifying and clustering said data. For example, a common task for neural networks is object recognition. Object recognition is when a neural network is given a large number of similar objects (street signs, images of animals, etc.) to inspect and analyze. It then interprets what the objects are while learning to identify patterns within said objects, eventually figuring out how to categorize future content. Figure 2 shows a neural network.

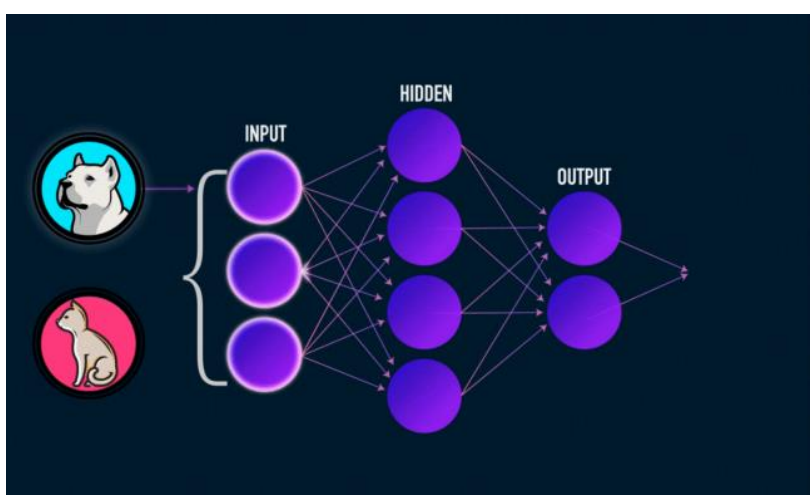

Figure 2: Neural Network (Source: www.analyticsindiamag.com)

Convolutional neural networks (CNN): Convolutional neural networks are a type of neural network specifically created for analyzing, classifying, and clustering visual imagery by using multilayer perceptrons. CNNs aid in object recognition within scenes (think: objects within a larger image, not just the standalone object) as well as digitized or handwritten text by using optical character recognition (OCR) tools

Generative adversarial networks (GAN): Generative adversarial networks are a type of neural network that can generate seemingly authentic photographs, at least on a superficial scale to human eyes. GAN-generated images take elements of photographic data and shape them into realisticlooking images of people, animals, and places. A recent example is presented in a paper by NVIDIA, a Style-Based Generator Architecture for GANs (StyleGAN). StyleGAN is capable of producing artificial imagery in a gradual fashion, from a pixelated, low-quality image that eventually grows into a realistic high-resolution image of an individual. The StyleGAN modifies features of what a person (or a cat) would look like, borrowing from actual images of existing people and cats, intricately assigning features and physical 
properties to a high level of detail (e.g. skin color, pores, hairstyle, eye color, facial hair, and more.)

Natural language processing (NLP): Natural language processing (NLP) helps computers process, interpret, and analyze human language and its characteristics by using natural language data. NLP is used with the intent of helping close the gap between humans and computers conversing with and understanding each other. An example of NLP can be seen in speech-to-text conversion transcriptions of voicemails.

Table 1: Major AI domains [9]

\begin{tabular}{|c|c|}
\hline Major AI domains & Description \\
\hline Large-Scale Machine learning & $\begin{array}{c}\text { Design of learning algorithms, as well } \\
\text { as scaling existing algorithms, to } \\
\text { work with large data sets. }\end{array}$ \\
\hline Deep Learning & $\begin{array}{c}\text { Model composed of inputs such as } \\
\text { image or audio and several hidden } \\
\text { layers of sub-models that serve as } \\
\text { input for the next layer and ultimately } \\
\text { an output of activation function. }\end{array}$ \\
\hline Natural Language Processing \\
(NLP) & $\begin{array}{c}\text { Algorithms that process human } \\
\text { language input and convert it into } \\
\text { understandable representations. }\end{array}$ \\
\hline Collaborative System & $\begin{array}{c}\text { Models and algorithms to help } \\
\text { develop autonomous systems that can } \\
\text { work collaboratively with other } \\
\text { systems and with humans. }\end{array}$ \\
\hline Computer Vision (Image & $\begin{array}{c}\text { The process of pulling relevant } \\
\text { Analytics) }\end{array}$ \\
& $\begin{array}{c}\text { information from an image or sets of } \\
\text { images for advanced classification } \\
\text { and analysis. }\end{array}$ \\
\hline $\begin{array}{c}\text { Algorithmic Game Theory and } \\
\text { Computational Social Choice }\end{array}$ & $\begin{array}{c}\text { Systems that address the economic } \\
\text { and social computing dimensions of } \\
\text { AI, such as how systems can handle } \\
\text { potentially misaligned incentives, } \\
\text { including self-interested human } \\
\text { participants or firms,and the } \\
\text { automated AI-based agents } \\
\text { representing them. }\end{array}$ \\
\hline Soft Robotics (Robotic Process & Automation of repetitive tasks and \\
Automation) & $\begin{array}{c}\text { common processes such as customer } \\
\text { servicing and sales without the need } \\
\text { to transform existing IT system maps. }\end{array}$ \\
\hline &
\end{tabular}

\section{DEEP LEARNING PRINCIPLE}

Deep learning is a branch of machine learning that teaches computers to do what comes naturally to humans: learn from experience [10]. Deep learning is a particular kind of machine learning (Figure 3) that achieves great power and flexibility by learning to represent the world as nested hierarchy of concepts or abstraction [2]. Machine learning algorithms use computational methods to "learn" information directly from data without relying on a predetermined equation as a model. Deep learning is especially suited for image recognition, which is important for solving problems such as facial recognition, motion detection, and many advanced driver assistance technologies such as autonomous driving, lane detection, pedestrian detection, and autonomous parking.

Deep learning uses neural networks to learn useful representations of features directly from large amount of data. Neural networks combine multiple nonlinear processing layers, using simple elements operating in parallel and inspired by biological nervous systems. Deep learning models can achieve state-of-the-art accuracy in object classification, sometimes exceeding human-level performance and accuracy. Models can be trained using a large set of labeled data and neural network architectures that contain many layers, usually including some convolutional layers. Training these models is computationally intensive and training can be accelerated by using a high performance GPU. It consists of an input layer, several hidden layers, and an output layer. The layers are interconnected via nodes, or neurons, with each hidden layer using the output of the previous layer as its input [9].

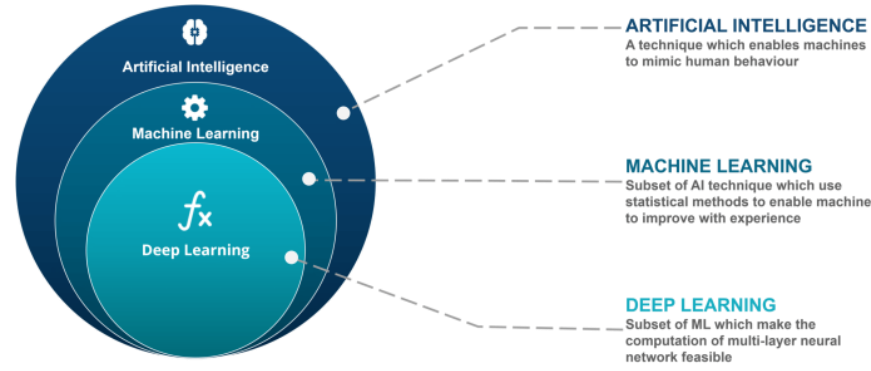

Figure 3: Relationship between AI, machine learning and deep learning [2]

\section{APPLICATIONS OF DEEP LEARNING FOR NATIONAL DEVELOPMENT}

Deep learning has wide application areas that are very important in national development. Some of the applications are stated by Aggarwal [11] as follow:

Self-Driving Cars - Deep Learning is the force that is bringing autonomous driving to life. A million sets of data are fed to a system to build a model, to train the machines to learn, and then test the results in a safe environment. The Uber AI Labs at Pittsburg is not only working on making driverless cars humdrum but also integrating several smart features such as food delivery options with the use of driverless cars. The major concern for autonomous car developers is handling unprecedented scenarios. A regular cycle of testing and implementation typical to deep learning algorithms is ensuring safe driving with more and more exposure to millions of scenarios. Data from cameras, sensors, geo-mapping is helping create succinct and sophisticated models to navigate through traffic, identify paths, signage, pedestrian-only routes, and real-time elements like traffic volume and road blockages.

News Aggregation and Fraud News Detection - There is now a way to filter out all the bad and ugly news from the news feed. Extensive use of deep learning in news aggregation is bolstering efforts to customize news as per readers. While this may not seem new, newer levels of sophistication to define reader personas are being met to filter out news as per geographical, social, economical parameters along with the individual preferences of a reader. Fraud news detection, on the other hand, is an important asset in today's world where the internet has become the primary source of all genuine and fake information. It becomes extremely hard to distinguish fake news as bots replicate it across channels automatically. The Cambridge Analytica is a classic example of how fake news, personal information, and statistics can influence reader perception (Bhartiya Janta Party vs Indian 
National Congress), elections (Read Donald Trump Digital Campaigns), and exploit personal data (Facebook data for approximately 87 million people was compromised). Deep Learning helps develop classifiers that can detect fake or biased news and remove it from your feed and warn you of possible privacy breaches. Training and validating a deep learning neural network for news detection is really hard as the data is plagued with opinions and no one party can ever decide if the news is neutral or biased.

Natural Language Processing (NLP) - Understanding the complexities associated with language whether it is syntax, semantics, tonal nuances, expressions, or even sarcasm, is one of the hardest tasks for humans to learn. Constant training since birth and exposure to different social settings help humans develop appropriate responses and a personalized form of expression to every scenario. NLP through Deep Learning is trying to achieve the same thing by training machines to catch linguistic nuances and frame appropriate responses. Document summarization is widely being used and tested in the Legal sphere making paralegals obsolete. Answering questions, language modeling, classifying text, twitter analysis, or sentiment analysis at a broader level are all subsets of natural language processing where deep learning is gaining momentum. Earlier logistic regression or SVM were used to build time-consuming complex models but now distributed representations, convolutional neural networks, recurrent and recursive neural networks, reinforcement learning, and memory augmenting strategies are helping achieve greater maturity in NLP. Distributed representations are particularly effective in producing linear semantic relationships used to build phrases and sentences and capturing local word semantics with word embedding (word embedding entails the meaning of a word being defined in the context of its neighboring words).

Virtual Assistants - The most popular application of deep learning is virtual assistants ranging from Alexa to Siri to Google Assistant. Each interaction with these assistants provides them an opportunity to learn more about your voice and accent, thereby providing you a secondary human interaction experience. Virtual assistants use deep learning to know more about their subjects ranging from your dine-out preferences to your most visited spots or your favorite songs. They learn to understand your commands by evaluating natural human language to execute them. Another capability virtual assistants are endowed with is to translate your speech to text, make notes for you, and book appointments. Virtual assistants are literally at the beck-and-call as they can do everything from running errands to auto-responding to specific calls to coordinating tasks between one and other team members. With deep learning applications such as text generation and document summarizations, virtual assistants can assist in creating or sending appropriate email copy as well.

Entertainment (VEVO, Netflix, Film Making, Sports Highlights, etc.) - Deep learning helps to minimize efforts of producers in actor selections etc. and also cut down cost of production. Wimbledon 2018 used IBM Watson to analyze player emotions and expressions through hundreds of hours of footage to auto-generate highlights for telecast. This saved them a ton of effort and cost. Thanks to Deep Learning, they were able to factor in audience response and match or player popularity to come up with a more accurate model (otherwise it would just have highlights of the most expressive or aggressive players) with the help of deep learning technology. Netflix and Amazon are enhancing their deep learning capabilities to provide a personalized experience to its viewers by creating their personas factoring in show preferences, time of access, history, etc. to recommend shows that are of liking to a particular viewer. VEVO has been using deep learning to create the next generation of data services for not only personalized experiences for its users and subscribers, but also artists, companies, record labels, and internal business groups to generate insights based on performance and popularity. Deep video analysis can save hours of manual effort required for audio/video sync and its testing, transcriptions, and tagging. Content editing and autocontent creation are now a reality thanks to Deep Learning and its contribution to face and pattern recognition. Deep Learning $\mathrm{AI}$ is revolutionizing the filmmaking process as cameras learn to study human body language to imbibe in virtual characters.

Visual Recognition - Imagine yourself going through a plethora of old images taking you down the nostalgia lane. You decide to get a few of them framed but first, you would like to sort them out. Putting in manual effort was the only way to accomplish this in the absence of metadata. The maximum you could do was sort them out based on dates but downloaded images lack that metadata sometimes. In comes, Deep Learning and now images can be sorted based on locations detected in photographs, faces, a combination of people, or according to events, dates, etc. Searching for a particular photo from a library requires state-of-the-art visual recognition systems consisting several layers from basic to advanced to recognize elements. Large-scale image Visual recognition through deep neural networks is boosting growth in this segment of digital media management by using convolutional neural networks, Tensorflow, and Python extensively.

Fraud Detection - Another domain benefitting from Deep Learning is the banking and financial sector that is plagued with the task of fraud detection with money transactions going digital. Autoencoders in Keras and Tensorflow are being developed to detect credit card frauds saving billions of dollars of cost in recovery and insurance for financial institutions. Fraud prevention and detection are done based on identifying patterns in customer transactions and credit scores, identifying anomalous behavior and outliers. Classification and regression machine learning_techniques and neural networks are used for fraud detection. While machine learning is mostly used for highlighting cases of fraud requiring human deliberation, deep learning is trying to minimize these efforts by scaling efforts.

Healthcare - According to NVIDIA, From medical imaging to analyzing genomes to discovering new drugs, the entire healthcare industry is in a state of transformation and GPU computing is at the heart. GPU-accelerated applications and 
systems are delivering new efficiencies and possibilities, empowering physicians, clinicians, and researchers passionate about improving the lives of others to do their best work. Helping early accurate and speedy diagnosis of lifethreatening diseases, augmented clinicians addressing the shortage of quality physicians and healthcare providers, pathology results and treatment course standardization, and understanding genetics to predict future risk of diseases and negative health episodes are some of the Deep Learning projects picking up speed in the Healthcare domain. Readmissions is a huge problem for the healthcare sector as it costs tens of millions of dollars in cost. But with the use of deep learning and neural networks, healthcare giants are mitigating health risks associated with readmissions while bringing down the costs.

Personalizations - Every platform is now trying to use chatbots to provide its visitors with personalized experiences with a human touch. Deep Learning is empowering efforts of e-commerce giants like Amazon, E-Bay, Alibaba, etc. to provide seamless personalized experiences in the form of product recommendations, personalized packages and discounts, and identifying large revenue opportunities around the festive season. Even recce in newer markets is done by launching products, offerings, or schemes that are more likely to please the human psyche and lead to growth in micro markets. Online self-service solutions are on the rise and reliable workflows are making even those services available on the internet today that were only physically available at one time.

Detecting Developmental Delay in Children - Speech disorders, autism, and developmental disorders can deny a good quality of life to children suffering from any of these problems. An early diagnosis and treatment can have a wonderful effect on the physical, mental, and emotional health of differently-abled children. Hence, one of the noblest applications of deep learning is in the early detection and course-correction of these problems associated with infants and children. This is a major difference between machine learning and deep learning where machine learning is often just used for specific tasks and deep learning on the other hand is helping solve the most potent problems of the human race.

\section{CONCLUSION AND RECOMMENDATION}

Most developing nations are very rich in terms of natural resources and also human population but the inability to utilize the resources objectively has been an issue. This has been attributed to poor management schemes. As a result most of the developing nations are facing development challenges such as health, education, fraud, mismanagement of public fund, drug and food counterfeiting, energy, Banking, security, transportation etc. AI has been used in many sectors in the developed world to improve performance of systems and also to solve some complex problems. Since machine learning has been used to address some complicated and complex health issues such as Ebola virus, therefore deep learning which is higher in accuracy and performance and also more potent in dealing with highly complex situations can be more useful to solve most the these development challenges disrupting national development. Thus, governments in the developing nations should make policies to implement AI deep Learning in every sector.

\section{REFERENCES}

[1] C., Gulcehre, "Welcome to Deep Learning", 2015, Retrieved on April 2, 2019 from: http://deeplearning.net/

[2] A., Atul, "AI vs Machine Learning vs Deep Learning", 2019 Retrieved on May 20, 2019 from: https://www.edureka.co/blog/aivs-machine-learning-vs-deep-learning/

[3] J.P.,Metzer, "The impact of artificial intelligence on international trade", 2019, The Brookings Institute

[4] C., Prince, Role of AI in developing countries', 2018 , Banking Sector, Retrieved on April 4, 2019 from: https://towardsdatascience.com/role-of-ai-in-developing-countriesbanking-sector-54a5e99d4bf

[5] J., Schmidhuber, "Deep Learning in Neural Networks: An Overview", Technical Report IDSIA, 2014, Pp. 1-88

[6] N., Wolchover, "New Theory Cracks Open the Black Box of Deep Learning", 2017, Retrieved on April 4, 2019 from: https://www.quantamagazine.org/new-theory-cracks-open-the-blackbox-of-deep-learning-20170921/

[7] B.J., Copeland, "Artificial Intelligence", 2019, Retrieved on April 4 2019 from: https://www.britannica.com/technology/artificialintelligence

[8] R., Reynoso, 11 Applications of Artificial Intelligence Today, G2 Crowd Inc. 2019, Retrieved on April 4, 2019 from: https://learn.g2.com/applications-of-artificial-intelligence

[9] T., Wang and P., Ngernlim, Artificial Intelligence in Asia and the Pacific, United Nations, ESCAPE, 2017

[10] Mathworks, "Deep Learning in Matlab", 2019, Retrieved on May 22, 2019 from: https://www.mathworks.com/help/deeplearning/ug/deep-learning-inmatlab.html

[11] S., Aggarwal, "Top 10 Applications of Deep Learning”, 2018, Retrieved on May 29, 2019 from: https://www.greatlearning.in/blog/top-10-applications-of-deeplearning/ 